Case Report

\title{
Mesenchymal chondrosarcoma in the mandible: report of a case with cytological findings
}

\author{
Adonai P. Cheim Jr, Thiago L. Queiroz, Whewel M. Alencar, Rodrigo M. Rezende \\ and Eneida F. Vencio
}

Department of Oral Pathology, School of Dentistry, Federal University of Goiás, Goiania, Brazil

(Received 12 October 2010 and accepted 9 March 2011)

\begin{abstract}
Mesenchymal chondrosarcoma is an infrequent malignancy of bone and soft tissue, characterized by its peculiar bimorphic histological pattern. The use of fine-needle aspiration (FNA) in the diagnosis of bone tumors is controversial. A 31-yearold woman presented with a mandibular lesion detected on routine examination for orthodontic treatment. Radiography revealed an ill-defined mixed radiolucency in the premolar region of the right mandible with invasive characteristics such as root resorption and widening of the periodontal ligament space of neighboring teeth. Blood clots obtained at FNA were fixed in formalin and exhibited spindle cells surrounding islands of pleomorphic chondroblasts against a bloody background. Histopathologically, clusters of spindle cells juxtaposed with mesenchymal tissue were detected, with a large hemangiopericytomatous component. In the present case, cytological findings combined with clinical and radiological data provided valuable information in establishing the diagnosis of malignancy and in planning further procedures and treatment. $(J$ Oral Sci 53, 245-247, 2011)
\end{abstract}

Keywords: blood clot; fine needle aspiration biopsy; hemangioperiocytoma pattern; mandible; mesenchymal chondrosarcoma.

Correspondence to Dr. Eneida Franco Vencio, Department of Oral Pathology, School of Dentistry, Federal University of Goiás, Praça Universitária, s/n Sala 2018 Setor Universitário, Goiania, GO, Brazil

Tel: +55-62-3209-6327

Fax: +55-62-3521-1886

Email: vencio56@hotmail.com

\section{Introduction}

Mesenchymal chondrosarcoma (MC) is an infrequent neoplasm involving bone and soft tissue. It represents approximately $1 \%$ of all chondrosarcomas (1). Described in 1959 (2), MC is characterized by a biphasic pattern consisting of areas of hyaline cartilage mixed with small cell malignancy. The relative amount of cartilage and the hemangiopericytomatous pattern vary considerably. Few cases have been reported in the jaw bones, and those that do occur in this region are usually seen in younger people (second to third decades), equally affecting both genders and both bones of the jaw $(3,4)$. MC of the jaw demonstrates indolent behavior compared with lesions from other anatomic locations, with average 5-year and 10-year survival rates of $82 \%$ and $56 \%$, respectively, in contrast with $54.6 \%$ to $27.3 \%$ for MC in general (3). No difference is described in the histological features between the indolent and rapid clinical course (10).

Efficiency of fine needle aspiration (FNA) biopsy in the diagnose of mesenchymal chondrosarcoma has been demonstrated by detecting cytological findings against a background of basophilic extracellular matrix (6). The material aspirated from the lesion has a high blood content and can be processed as a tissue biopsy (9). In the present case, formalin-fixed blood clots from FNA biopsy were suspicious of malignancy. This procedure, together with the clinical presentation and radiographic features, provided valuable information for planning further surgical procedures and treatment.
\end{abstract}

\section{Case Report}

A 31-year-old woman was referred for examination of a mandibular lesion detected on routine examination for 
orthodontic treatment. No abnormality was apparent on clinical examination. Radiography (panoramic and periapical) revealed an ill-defined radiolucency in the premolar region of the right mandible; the lesion exhibited invasive characteristics such as root resorption and widening of the periodontal ligament space of neighboring teeth (Figs. 1 and 2). Based on the clinical and radiographic findings, FNA biopsy was conducted. Cytological smears consisted mostly of blood cells and a few spindle cells showing pleomorphic changes (Fig. 3A). When the blood clot obtained at FNA was fixed in formalin and processed, it revealed islands of spindle cells, surrounded by mesenchymal cells and a large hemangiopericytomatous component. Aspirated cells also showed hyperchromatic nuclei (Fig. 3B). Chondroid islands were also seen.

We were suspicious of chondroid malignancy on the basis

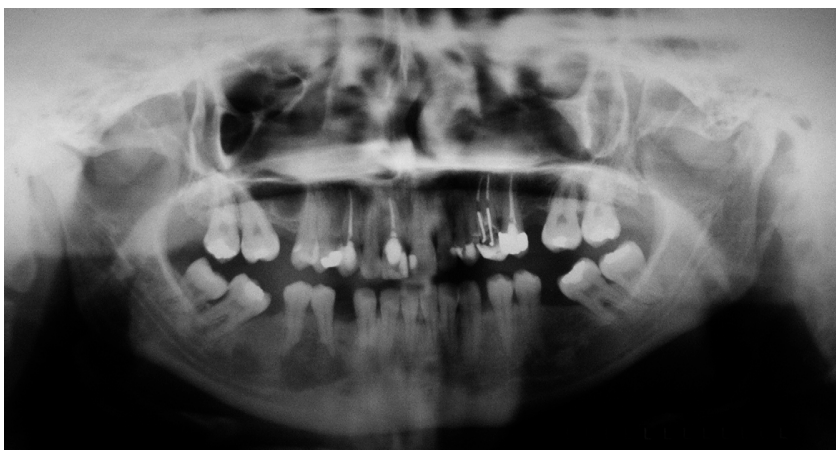

Fig. 1 Panoramic radiography revealing an ill-defined heterogeneous radiolucency between the premolar teeth in the right mandible. Note root resorption of neighboring teeth.

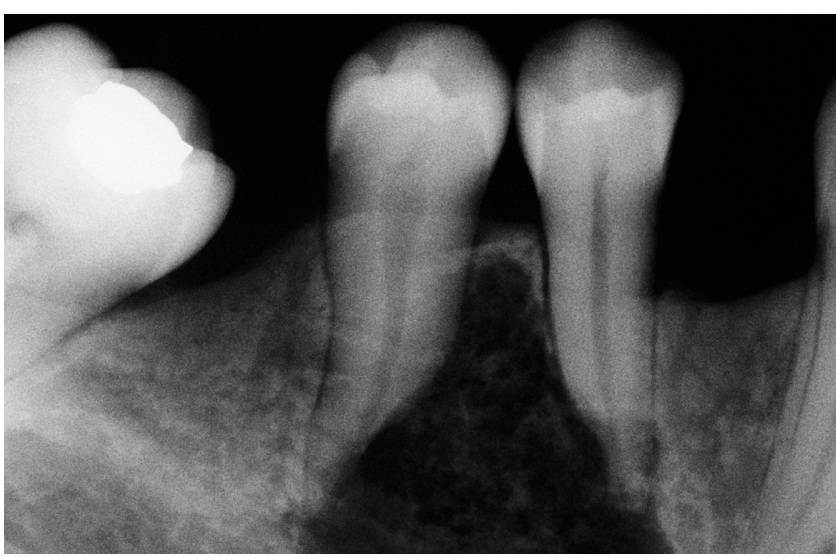

Fig. 2 Periapical radiography showing root resorption in more detail and widening of the periodontal ligament space of neighboring teeth. Note the absence of the distal lamina dura in the right mandibular in both premolars teeth. of the cytological findings and clinical and radiographic data. An incisional biopsy was therefore performed, which revealed a bimorphic pattern with focal areas of relatively mature cartilage formation and other areas of highly cellular tissue composed of small round or spindle-shaped cells (Fig. 3C). A prominent hemangiopericytoma-like pattern with numerous capillary channels was evident. Areas of calcification were also identified. A diagnosis of mesenchymal chondrosarcoma was established. The patient underwent radical resection of the mandible. The resected tumor specimen showed predominant cartilaginous component. No tumor tissue was detected in regional lymph nodes. The patient is free of disease 3 years after treatment.

\section{Discussion}

The use of FNA in the diagnosis of bone tumor is controversial. Here we have presented a case of MC with cytological findings. Blood clots obtained at FNA were fixed in formalin and processed as for tissue biopsies, revealing cytological findings suspicious of chondroid
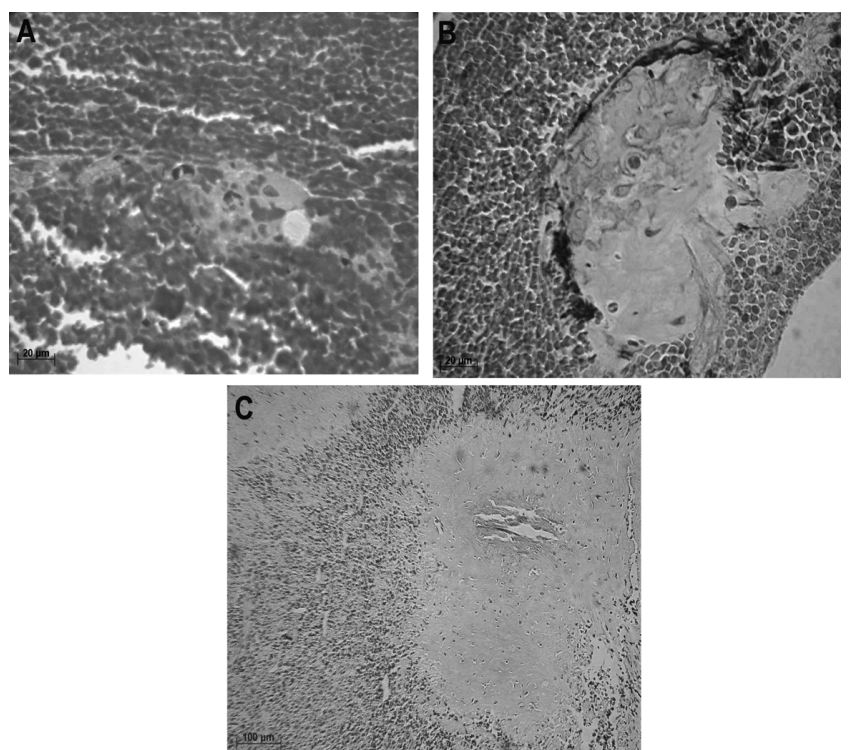

Fig. 3 Formalin-fixed blood clots and tissue biopsy of mesenchymal chondrosarcoma. A, Fine-needle aspiration smear of a few spindle cells against a bloody background (hematoxylin-eosin stain; original magnification $\times 400$ ). B, Aspirated cells showing pleomorphic changes were surrounded by spindle cells with osteo-cartilaginous metaplasia. $\mathrm{C}$, Tissue fragment from incisional biopsy showing a bimorphic pattern composed of lobules of cartilage and sheets of mesenchymal tissue. Note the numerous undifferentiated small cells with hemangiopericytoma-like arrangement and mineralization. 
malignancy. First described in 1973 (8), FNA is a safe, costeffective procedure, and provides reliable information in the diagnosis of various solid tissue neoplasms. However, its role in the diagnosis of bone tumors remains controversial (6). Some studies have noted that FNA has high sensitivity and specificity in the diagnosis of bone neoplasms (7), whereas others have shown that it is more effective in the diagnosis of recurrence or metastasis (5). It is considered to have high diagnostic accuracy when used in conjunction with radiographic and clinical findings $(6,7)$.

In the current case, a young woman was referred for examination of a mandibular lesion. Radiography revealed an ill-defined mixed radiolucency in the right mandible with features of invasive behavior such as root resorption and widening of the periodontal ligament space of neighboring teeth. Processing of the blood clots as tissue biopsies revealed islands of pleomorphic cells arranged in nodules and showing osteo-cartilaginous differentiation as well as cells with hyperchromatic nuclei. Histopathological findings showed a bimorphic pattern composed of undifferentiated spindle cells and well-defined nodules of cartilaginous tissue with a hemangiopericytomatous pattern.

In the present case, cytological findings from formalinfixed blood clots together with clinical and radiographic data suggested a chondroid malignancy in the mandible. Although the use of FNA for diagnosis of solid bone tumors is restricted, processing of blood clots in the present case combined with clinical and radiological data provided valuable information in establishing the diagnosis of malignancy and in planning further procedures and treatment.

\section{Acknowledgments}

The authors would like to thank Dr. Krishman K. Unni from the Mayo Clinic, Rochester, Minnesota, USA, for reviewing this case.

\section{References}

1. Fletcher CDM (2000) Diagnostic histopathology of tumors. 2nd ed, Churchill Livingstone, London, 1562-1563.

2. Lichtenstein L, Bernstein D (1959) Unusual benign and malignant chondroid tumors of bone. A survey of some mesenchymal cartilage tumors and malignant chondroblastic tumors, including a few multicentric ones, as well as many atypical benign chondroblastomas and chondromyxoid fibromas. Cancer 12, 1142-1157.

3. Vencio EF, Reeve CM, Unni KK, Nascimento AG (1988) Mesenchymal chondrosarcoma of the jaw bones: clinicopathologic study of 19 cases. Cancer 82, 2350-2355.

4. Takahashi K, Sato K, Kanazawa H, Wang XL, Kimura T (1993) Mesenchymal chondrosarcoma of the jaw: report of a case and review of 41 cases in the literature. Head Neck 15, 459-464.

5. Dodd LG (2006) Fine-needle aspiration of chondrosarcoma. Diagn Cytopathol 34, 413-418.

6. Trembath DG, Dash R, Major NM, Dodd LG (2003) Cytopathology of mesenchymal chondrosarcomas: a report and comparison of four patients. Cancer 99 , 211-216.

7. Bommer KK, Ramzy I, Mody D (1997) Fine-needle aspiration biopsy in the diagnosis and management of bone lesions: a study of 450 cases. Cancer 81, 148-156.

8. Stormby N, Akerman M (1973) Cytodiagnosis of bone lesions by means of fine-needle aspiration biopsy. Acta Cytol 17, 166-172.

9. Althoff CE, Hermann KGA, Wiechen K, Lembcke A, Enzweiler CNH, Hamm B, Rogalla P (2006) Formalin-fixed blood clots - additional histological findings on computed tomography-guided fineneedle aspiration biopsies in comparison with core biopsies. J Comput Assist Tomogr 30, 386-390.

10. Unni KK, Inwards CY (2009) Dahlin's bone tumors: general aspects and data on 10,165 cases. 6th ed, Lippincott Williams \& Wilkins, Philadelphia, 9397. 